\title{
Energy localization and transport in two-dimensional electrical lattices
}

\author{
L.Q. English ${ }^{1}$, F. Palmero ${ }^{2}$, J.F. Stormes ${ }^{1}$, J. Cuevas ${ }^{2}$, R. Carretero-González ${ }^{3}$, P.G. Kevrekidis ${ }^{4}$ \\ ${ }^{1}$ Department of Physics and Astronomy, Dickinson College, Carlisle, PA 17013, USA \\ 2 Departamento de Física Aplicada I, Universidad de Sevilla, Avda. Reina Mercedes, 41012-Sevilla, Spain \\ ${ }^{3}$ Department of Mathematics and Statistics, San Diego State University, San Diego, CA 92182, USA \\ ${ }^{4}$ Department of Mathematics and Statistics, University of Massachusetts, Amherst, MA 01003, USA
}

\begin{abstract}
Intrinsic localized modes (ILMs) have been generated and characterized in two-dimensional nonlinear electrical lattices which were driven by a spatially-uniform voltage signal. These ILMs were found to be either stationary or mobile, depending on the details of the lattice unit-cell, as had already been reported in one-dimensional lattices; however, the motion of these ILMs is qualitatively different in that it lacks a consistent direction. Furthermore, the hopping speed seems to be somewhat reduced in two dimensions due to an enhanced Peierls-Nabarro (PN)barrier. We investigate both square and honeycomb lattices composed of $6 \times 6$ elements. These direct observations were further supported by numerical simulations based on realistic models of circuit components. The numerical study moreover allowed for an analysis of ILM dynamics and pattern formation for larger lattice sizes.
\end{abstract}

\section{Introduction}

Solitons emerge as classes of solutions to many nonlinear differential equations described chiefly by one spatial dimension; prominent examples are the $\mathrm{KdV}$ equation, the sine-Gordon or the nonlinear Schrödinger equations. In two dimensions, quasi onedimensional localization patterns can often still occur [1], but robust two-dimensional (2D) localization in continuous media is rather atypical (see Ref. [2] and references therein). However, it is well-known that discreteness of the underlying medium can help to stabilize such localized solutions even in higher dimensions $[3,4]$.

ILMs have been considered experimentally in a variety of other settings including (but not limited to) micromechanical cantilever arrays [5], Josephsonjunction ladders (JJLs) [6], layered antiferromagnetic crystals [7], halide-bridged transition metal complexes [8], and dynamical models of the DNA double strand [9]. Yet, in most of these examples, the coherent structures are effectively one-dimensional.

Even in higher dimensional lattice settings (e.g. in optical waveguide arrays or photorefractive crystals
[10]), however, it is often difficult to find stable localized eigenmodes experimentally, and the states are typically stationary. The few experimental results so far have been in nonlinear optics and atomic physics [11] settings where stable localized solitary wave structures have been reported.

Here we show experimentally the existance and stability of intrinsic localized modes (ILMs), or discrete breathers (DBs) in two-dimensional, damped-driven electrical lattices (see also Ref. [12]). We characterize these breather states in parameter space, and we compare to numerical simulations and stability analysis. Whereas in the experiment, the lattice size was fixed at $6 \times 6$, in the numerical simulations larger lattices were also explored. Finally, we focus particularly on versions of these lattices that support moving breathers. As we will see, the ILM motion in 2D differs from that in $1 \mathrm{D}$.

\section{The system}

The electical 2D lattices investigated in this study can be visualized as follows: imagine two planes; the top plane is regularly divided into squares or honeycombs (both types of lattices were studied), and the bottom plane represents electrical ground. Within the top plane, each edge represents a coupling inductor $\left(L_{1}=680 \mu \mathrm{H}\right)$, and each vertex represents a lattice node to which a driven, nonlinear RLC-resonator is attached. The other end of these RLC-resonators connect to the bottom plane, i.e. the ground. In Fig. 1, the left two panels illustrate the top plane (when extended), and the right panel illustrates the nonlinear RLC-resonator. It is comprised of a varactor diode (NTE 618) characterized by a nonlinear capacitance $C(V)$ and an inductor $L_{2}=330 \mu \mathrm{H}$. Point $\mathrm{A}$ is driven by a single sinusoidal voltage source $V(t)$ via a resistor $R=10 \mathrm{k} \Omega$, with amplitude $V_{d}$ and frequency $f$. It is important to understand that every node is driven by the same voltage signal. We study two different unit cell versions, with the only difference being the presence of a block capacitor $(1 \mu \mathrm{F})$ between the diode and the coupling inductor in one of them, as explained in 


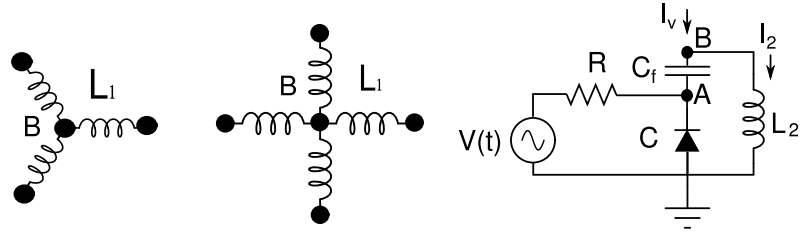

Figure 1: Left: Schematic circuit diagrams of the basic geometry of the two electrical transmission lines (honeycomb and square), where black points represent single elements (right), with or without the block capacitor $C_{f}$. Every single element is connected to the same sinusoidal voltage source $V(t)$ via a resistor $R$, and grounded.

Ref. [13]. The effect of the block capacitor is to make ILMs mobile.

In the experiment, the lattice size was fixed at 6 by 6 , resulting in 36 elements, with periodic boundary conditions. Node voltages were digitized at points $B$ at a rate of $2.5 \mathrm{MHz}$ using a multichannel analog-todigital converter. Periodic boundary conditions were instituted by connecting each boundary element with its corresponding one on the other side of the lattice via inductors $L_{1}$.

Using basic circuit theory, and the realistic modeling of circuit components, the dynamics of the lattice (including the block capacitors) can be formulated in terms of a set of coupled differential equations. References [14] and [12] give more details.

\section{Results}

Figure 2(a) shows the basic steady-state result in a square lattice without block capacitors, where the driver frequency and amplitude was chosen at 290 $\mathrm{kHz}$ and $1.75 \mathrm{~V}$, respectively. The gray-scale in the graph indicates a measure of energy in the electrical oscillation, obtained from the data by simply squaring the voltage and averaging over one period. As is evident, one driven ILM is generated in this lattice centered at node $(3,2)$. Nearest neighbor oscillations are also still clearly enhanced, but beyond that the energy quickly fades into the background excitation. The ILM energy-profile is illustrated in panel (b), which shows both $\mathrm{x}$ - (black, filled circles) and y-direction (red, open circles) cuts through the ILM center. The energy concentrated at the site of the ILM center is seen to be roughly six times as large as that found in the wings. The way that this ILM is experimentally produced becomes clear when focusing on the transients immediately after the driver is turned on: the driver initiates a modulational instability that quickly breaks up the uniform mode in favor of localized excitations - a common route to energy localization in lattices $[15,7]$.
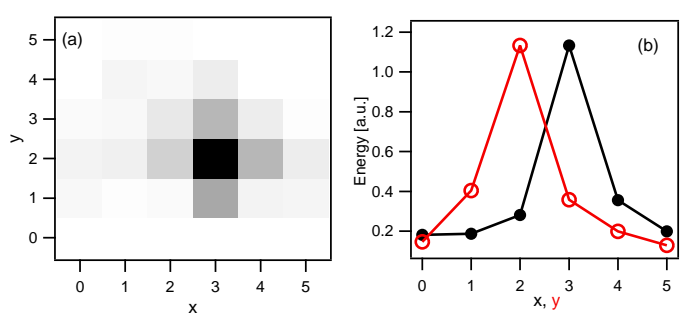

Figure 2: Localized distribution of energy in a $6 \times 6$ square lattice in response to spatially uniform driving. (a) Density plot with grayscale indicating power at each node, and (b) Cuts through the ILM center in the $\mathrm{x}$ - and $\mathrm{y}$-direction (black filled and red open circles, respectively).

Next, let us turn to a very similar lattice with the only modification being that block capacitors are included. From 1D results, we would expect that this inclusion now allows for mobility [13]. Indeed, as illustrated in Fig. 3(a) and (b), we now observe the ILM hopping from site to site throughout the lattice - here from site $(4,0)$ to $(4,1)$. At each site the ILM lingers for a certain duration (on the order of $100 \mu \mathrm{s}$ ) as it struggles to overcome the well-known Peierls-Nabarro $(\mathrm{PN})$ barrier [3]. Finally, it is forced away from the node via a mechanism that is very similar to the one reported in the $1 \mathrm{D}$ lattice [13]. Specifically, the presence of the large nonlinear oscillation at the ILM center results in a gradual charging of the block capacitor; the charge on the capacitor, in turn, creates a voltage which reverse-biases the diode. The end effect is that the ILM gradually creates a local impurity in the lattice which then repels the ILM.

In Fig. 3(c), the energy at both nodes is plotted as a function of time. The oscillations seen in the energy are due to incomplete smoothing over one period. We observe the gradual buildup of energy in node $(4,1)$ over a duration of roughly $60 \mu \mathrm{s}$. Then, as soon as the amplitude at both nodes has approximately equalized, there ensues a rapid drop-off of energy in node $(4,0)$, and the ILM has completed its transition to $(4,1)$. The process is then repeated at a later time for node $(4,1)$. It is interesting to note the asymmetry between the build-up and release of energy as the ILM enters and leaves a particular node.

Nevertheless, and contrary to the simpler situation in $1 \mathrm{D}$, where a clear direction of movement arises, the motion through the 2D lattice appears to be more complex. Note that given the small size of the lattice, in addition to the role of inhomogeneities, small amplitude residual excitations ("phonons") are also important in directing the breather motion.

Despite the complex nature of the motion of the 

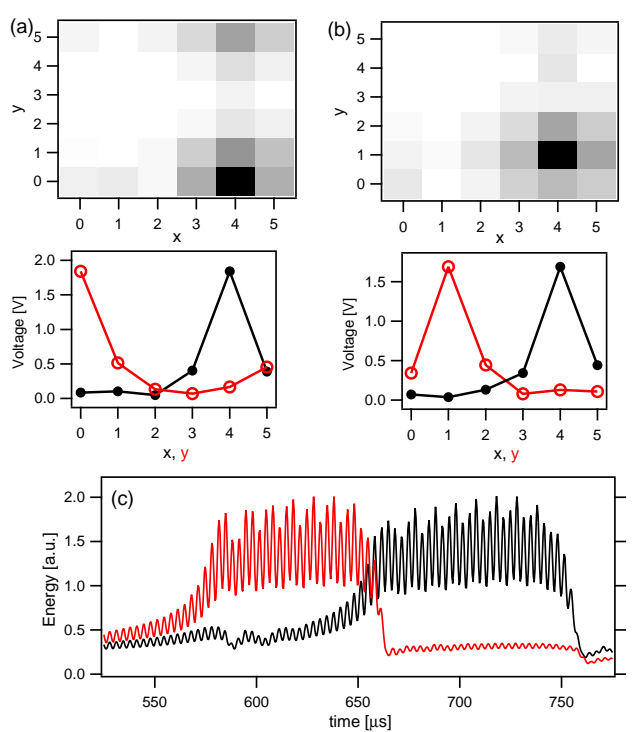

Figure 3: The square lattice with block capacitors. $f=300 \mathrm{kHz}, V_{d}=1.75 \mathrm{~V}$. Top: The voltage at each node is plotted in grayscale at the time within one cycle of maximum ILM amplitude. Bottom: the voltage profiles of the ILM. (a) and (b) show two snapshots separated in time by $\Delta t=80 \mu \mathrm{s}$. (c) The energy at both nodes as a function of time.

breathers, an organizing principle obeyed in both one and two dimensions is that the ILM never hops back to the node it occupied prior to its current location due to some residual capacitor-charge impurity associated with that node. In the topologically restricted one dimensional case, this principle necessarily gives rise to orderly, uni-directional motion. In two-dimensional hexagonal lattices there are still two choices available to the ILM, and in a square lattice there are three. Thus, the motion does not have to be uni-directional.

One interesting comparison we can now examine is the PN-barrier against breather motion in $1 \mathrm{D}$ and $2 \mathrm{D}$ lattices. In 1D, Ref. [16] reported detailed measurement of ILM speed. It was found that the slowest a single ILM could move in a 1D lattice of 24 nodes was 15 nodes per millisecond $(15 \mathrm{a} / \mathrm{ms})$. At those driving conditions, the ILM center attained a maximum positive voltage of $2 \mathrm{~V}$. From Fig. 3 it is clear that the maximum voltage-amplitude at the ILM center was also $2 \mathrm{~V}$ in the $2 \mathrm{D}$ lattice data shown. Since the ILM speed should be primarily a function of center amplitude [13], we should be able to compare the speeds directly.

In the 2D square lattice, we observe 42 hopping events within a $4 \mathrm{~ms}$ time span. This corresponds to an average hopping speed of $10.5 \mathrm{a} / \mathrm{ms}$. As is also true in $1 \mathrm{D}$, the time that an ILM spends at a particular lattice site can vary due to inhomogeneities in the experimental lattice, and so we would expect some uncertainty
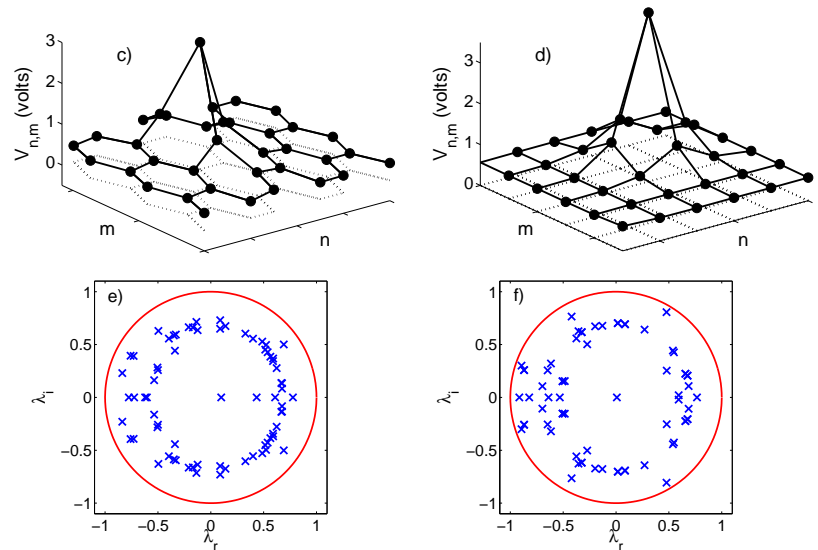

Figure 4: Numerical profiles of a stationary breather in a honeycomb lattice (left), and a square lattice (right) in the $(n, m)$ plane. The bottom panels show the Floquet numerical linearization spectrum corresponding to the honeycomb lattice and the square lattice, respectively, confirming the stability of solutions. The driver has amplitude $V_{d}=1.5 \mathrm{~V}$ and frequency $f=283 \mathrm{kHz}$ in the honeycomb lattice case, and $V_{d}=2$ $\mathrm{V}$ and $f=301 \mathrm{kHz}$ in the square lattice case

in these numbers. Nevertheless, since we are averaging over 42 hopping events, we don't expect a large uncertainty in these numbers, certainly no larger than $\sqrt{42} \cong 6.5$; thus, we would not expect values larger than $42+6.5 / 2=45.25$, which is still significantly below the 60 observed in 1D. Therefore, the data appears to indicate that the $\mathrm{PN}$-barrier is enhanced by going from one to two lattice dimensions.

In order to find ILMs in our theoretical model, we have employed the experimental results as seeds for a shooting method [17] in order to find spatially localized periodic orbits in the governing differential equations. Once the exact numerical solutions are identified (up to a prescribed tolerance), their stability properties are explored by means of a Floquet analysis [18].

In the case without block-capacitors, we obtain stationary and stable one-peak breathers, as shown in Fig. 4. These localized modes are robust, persisting as long as the driver remains on.

Numerics are generally found to be in good quantitiative agreement with experimental results [12]. Now that numerical work has confirmed the experimental results and also, according to the bottom panels of Fig. 4, established the breather stability in small lattices, we can use these simulations on larger lattices, where experimental data is missing due to the practical difficulty in constructing them. Figure 5 shows the stable numerical breather solutions in larger lattices without block capacitors for a $21 \times 21$ lattice with periodic boundary conditions; in a slightly larger lattice $(36 \times 36)$, the number of ILMs increases to two. 


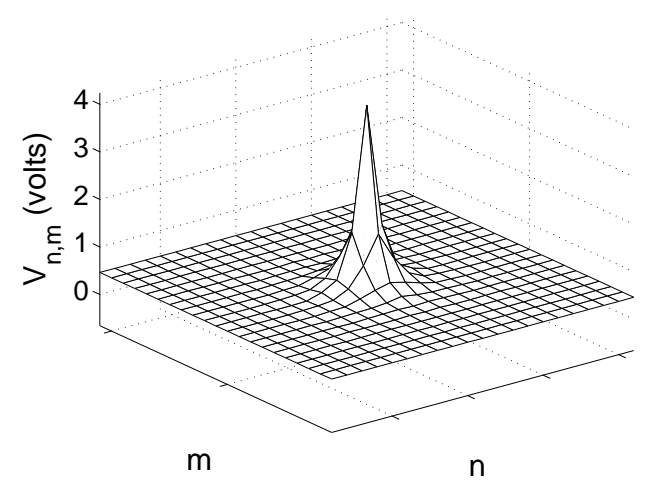

Figure 5: Numerical solution (stable) of a stationary breather in the larger $21 \times 21$ electrical lattice.

It should be noted that these breather solutions in the larger lattices cannot be continued as the lattice size is reduced to the solutions shown for instance in Fig. 4, and they do not connect in a straightforward way to the breather states seen before in $6 \times 6$ lattices. Rather, we find that these breather become unstable, and even disappear below a threshold lattice size. More work has to be done to elucidate the relations between the stable breathers in small lattices and their corresponding generalizations in larger lattices.

\section{Conclusion}

We have experimentally induced two-dimensional energy localization in damped-driven electrical lattices. This was done by first generating breathers via a modulational instability and then stabilizing them by continued direct driving. Within particular versions of these 2D electrical lattices, ILMs become mobile, and they were observed to meander in the lattice without an apparent directionality. Numerical simulations, which closely modeled the circuit elements comprising the unit cell, corroborated these findings and were able to extend them to larger lattices. Future directions include an exploration of possible vortex distribution of localized energy or phase, a fuller charcterization of localized solutions in larger 2D lattices and in lattices of different shape. This study also opens up the possibility of analyzing in greater detail breather-breather and breather-impurity interactions; another extension of interest would be the introduction of spatial anisotropy into the $2 \mathrm{D}$ lattice via the coupling inductors and the study its effect on ILM shape and motion.

\section{References}

[1] M.J. Ablowitz and D.E. Baldwin, Phys Rev. E 86, 036305 (2012).

[2] K. Staliunas, R. Herrero, G.J. de Valcárcel, Phys. Rev. A 75, 011604 (2007).

[3] S. Flach and A.V. Gorbach, Phys. Rep. 467, 1 (2008).

[4] P.G. Kevrekidis, The Discrete Nonlinear Schrödinger Equation, Springer-Verlag (Heidelberg, 2009).

[5] M. Sato, B.E. Hubbard, and A.J. Sievers, Rev. Mod. Phys. 78, 137 (2006)

[6] P. Binder, et al., Phys. Rev. Lett. 84, 745 (2000); E. Trías, J.J. Mazo, and T.P. Orlando, Phys. Rev. Lett. 84, 741 (2000);

J.J. Mazo and T.P. Orlando, Chaos 13, 733 (2003);

A.V. Ustinov, Chaos 13, 716 (2003).

[7] L.Q. English, M. Sato, and A.J. Sievers, Phys. Rev. B 67, 024403 (2003);

U.T. Schwarz, L.Q. English, and A.J. Sievers, Phys. Rev. Lett. 83, 223 (1999).

[8] B.I. Swanson, et al., Phys. Rev. Lett. 82, 3288 (1999).

[9] M. Peyrard, Nonlinearity 17, R1 (2004).

[10] F. Lederer, et al., Phys. Rep. 463, 1 (2008).

[11] J.W. Fleischer, et al., Nature 422, 147 (2003);

B. Eiermann, et al., Phys. Rev. Lett. 92, 230401 (2004).

[12] L.Q. English, F. Palmero, J.F. Stormes, J. Cuevas, R. Carretero-González, P.G. Kevrekidis, Phys. Rev. E, to appear (2013).

[13] L.Q. English, et al., Phys. Rev. E 81, 046605 (2010).

[14] F. Palmero, et al., Phys. Rev. E 84, 026605 (2011).

[15] J.L. Marín, F. Falo, P.J. Martínez, and L.M. Floría, Phys. Rev. E 63, 066603 (2001).

[16] L.Q. English, R. Basu Thakur, R. Stearrett, Phys. Rev. E 77, 066601 (2008).

[17] J.L. Marín and S. Aubry, Nonlinearity 9, 1501 (1996);

T. Cretegny and S. Aubry, Phys. Rev. B 55, R11929 (1997)

[18] S. Aubry, Physica D 261, 1 (1996) 